\title{
EFFECT OF CELLULOSIC POLYMER ON PHYSICO MECHANICAL PROPERTIES OF SUPERPOROUS HYDROGEL OF AN ANTIHYPERTENSIVE DRUG AND DRUG RELEASE KINETICS FROM IT
}

\author{
GOPA ROY BISWAS*, DEBOLINA ROY, SUTAPA BISWAS MAJEE
}

Division of Pharmaceutics, NSHM College of Pharmaceutical Technology, NSHM Knowledge Campus, Kolkata-Group of Institutions, 124 B.

L. Saha Road, Kolkata 700053, India

Email: gopa.biswas@nshm.com

Received: 29 Jun 2019, Revised and Accepted: 06 Aug 2019

\begin{abstract}
Objective: Super porous hydrogels (SPHs), a novel drug delivery system can be developed to retain drugs in the gastric medium. The aim of the present investigation was to prepare superporous hydrogels (SPHs) of Atenolol to release the drug in sustained manner in the gastric environment and study the effect of two grades of hydroxyl methyl cellulose along with Carbopol 971p on the physico mechanical properties and drug release
\end{abstract} kinetics of the formulations.

Methods: Superporous hydrogels of Atenolol were prepared with two grades of Hydroxy Propyl Methyl Cellulose (HPMC K100M and HPMC K15 M) along with Carbopol 971p the structural morphology of hydrogel was observed by Scanning Electron Microscopy. Study on Physico mechanical characteristics and drug release were done.

Results: Scanning Electron microscopy studies of the formulations revealed the presence of large number of pores in different size ranges like $1 \mu \mathrm{m}$, $2 \mu \mathrm{m}, 10 \mu \mathrm{m}$, confirming the formulations as superporous hydrogel. A correlation had been found between porosity, density and \% swelling index. The drug release data from the formulations obeyed Higuchi and Korsmeyer-Peppas kinetics. Further, the data were fitted to the Kopcha model for confirming drug release by a combination of diffusion-controlled and chain relaxation-swelling mechanism.

Conclusion: Among the six formulations, where HPMC K15 M and HPMC K100 M both were present, the gel became more hydrophobic and retarded the release of drug. From the drug release kinetics data, it can be concluded that the diffusion mechanism predominated the drug release process, leading to quasi diffusion and Fickian diffusion mechanism.

Keywords: Super porous hydrogel, Gastro retentive, Porosity, Higuchi, Korsmeyer-Peppas, Diffusion, Kopcha Kinetics

(C) 2019 The Authors. Published by Innovare Academic Sciences Pvt Ltd. This is an open access article under the CC BY license (http://creativecommons.org/licenses/by/4.0/) DOI: http://dx.doi.org/10.22159/ijap.2019v11i5.34734

\section{INTRODUCTION}

Hydrogels are crosslinked hydrophilic polymers with a network structure consisting of acidic, basic, or neutral monomers and they have the ability to absorb huge amount of water. The swelling properties of hydrogels are closely related to the factors like the elasticity of the network, the presence of hydrophilic functional groups (such as- $\mathrm{OH},-\mathrm{COOH},-\mathrm{CONH}_{2},-\mathrm{SO}_{3} \mathrm{H}$ ) in the polymer chains, the extent of cross linking, and porosity of the polymer. Hydrogel swell in water with some mechanical strength but their swelling index and mechanical strength are not sufficient to exhibit fast swelling properties [1]. In some cases, the slow swelling is beneficial but there are many situations where a fast swelling characteristic is more desirable. Therefore, a new generation of hydrogels, which swell and absorb water rapidly, has been developed. Examples of this new generation are superporous hydrogel, which swell to an equilibrium size in a short period of time [2]. The fast swelling and strong mechanical properties of SPHs make them highly useful for various pharmaceutical and biomedical applications [3]. Superporous hydrogels (SPHs) possess an average pore size of greater than 100 microns and swell in equivalent sizes within a very short period because of rapid intake of water by capillary wetting through a number of interconnected microscopic pores [4]. SHPs have a tendency to swell to a large size with a swelling ratio of about 100 or more and their mechanical strength [5] should be high enough to withstand the pressure as gastroretentive drug delivery. Due to the highly porous structure, SPHs has hundred time's greater surface area and shorter diffusion distance than conventional hydrogels do.

The drugs having a narrow absorption window, i.e. mainly absorbed from the proximal small intestine, bioavailability of those drugs can be well increased by gastric retention. For drugs which are absorbed rapidly from the gastrointestinal tract (GIT), should have slow release from the stomach to improve the bioavailability. Several important properties of SPHs, like fast swelling capacity, large swelling ratio, and surface slipperiness, make them an excellent candidate to develop gastric retention devices [6]. Even though these super porous hydrogels provided drastically fast swelling kinetics and high swelling degree, the mechanical strength of the fully swollen super porous hydrogels was besides poor to be useful. Hence mechanically strong super porous hydrogels can be made by increasing the cross linking density, but this would result in a very small extent of swelling with a loss of the superabsorbent property [7].

When applied as drug carriers, the highly swollen hydrogels remain in the stomach for a long time, releasing almost all loaded drugs, since their volumes are too big to transport through the pylorus and their sheer bulk obstruct their transport to the next organ via the narrow pylorus. This unique swelling property allows them to be used as gastric retention carriers providing a sustained release through long residence in the stomach [8]. To be used as an effective gastro retentive drug delivery, the hydrogels are required to possess not only fast swelling, but also following properties: biocompatibility, biodegradability, high swelling capacity, high mechanical strength, and stability in acidic condition [9].

The aim of the study was to develop superporous hydrogel of Atenolol, an antihypertensive drug which is highly unstable at basic $\mathrm{pH}$. Hence there was a need to develop a gastroretentive system on it so that the formulation would be confined in the stomach and release the drug in a sustained manner in the gastric environment. The superporous structure would swell up by absorbing water through capillary forces and its integrity would be maintained for a sufficient period.

\section{MATERIALS AND METHODS}

\section{Materials}

Atenolol was a gift sample from Windlas Biotech Ltd, Dehradun, India. HPMC were obtained from Colorcon Asia Pvt. Ltd and Carbopol 971p from Mylan Laboratories Ltd. A. P. India. Ac-Di-Sol was obtained from Matrix Laboratories, Hyderabad. Triethanolamine was purchased from 
Loba Chemie Pvt Ltd. All other ingredients used were of pharmaceutical grade.

\section{Drug-polymer interaction study}

Drug-excipient interaction, one of the most essential parameters, is studied before development of the formulations. Atenolol, polymers and polymer mix; with drug was mixed separately with IR grade $\mathrm{KBr}$ in the ratio 1:100 and corresponding pellets were prepared by applying 5.5 metric ton of pressure in a hydraulic press. Polymers were HPMC K100M, HPMC K15 M, Carbopol 971p. The pellets were scanned over a wave number range of 4000 to $400 \mathrm{~cm}-1$ in FTIR spectroscope (ALPHA T, Bruker, USA).

Table 1: Compositions of superporous hydrogel

\begin{tabular}{|c|c|c|c|c|c|c|}
\hline Ingredients & F1 & F3 & G1 & G2 & A1 & A3 \\
\hline HPMC K100M: HPMC K 15M: Carbopol 971p & $1: 0: 1$ & $1: 0: 2$ & $0: 1: 1$ & $0: 1: 2$ & $1: 1: 1$ & $1: 1: 2$ \\
\hline SodiumChloride $(\%)$ & 5 & 5 & 5 & 5 & 5 & 5 \\
\hline Ac-Di-Sol (\%) & 10 & 10 & 10 & 10 & 10 & 10 \\
\hline Atenolol (Mg) & 50 & 50 & 50 & 50 & 50 & 50 \\
\hline Triethano-Lamine(Drops) & 7 & 7 & 7 & 7 & 7 & 7 \\
\hline Double Distilled Water(Ml) & 20 & 20 & 20 & 20 & 20 & 20 \\
\hline
\end{tabular}

\section{Preparation of superporous hydrogels}

Hydrophilic polymers had been selected to prepare superporous hydrogel of Atenolol. The polymers were used in different ratios. HPMC (Hydroxy Propyl Methyl Cellulose) of different grades was first dispersed in double distilled water alone and also in combination of Carbopol 971p. In the meantime, the required amount of drug, Atenolol was mixed to it. The mixture was stirred in magnetic stirrer until thickening occurred and then neutralized by drop-wise addition of $50 \%(\mathrm{w} / \mathrm{w})$ triethylamine, until a transparent gel appeared. Quantity of tri ethanolamine was adjusted to prepare gel with desired $\mathrm{pH}$. Gels were stored for $24 \mathrm{~h}$ at the room temperature to stabilize [10] (table 1).

\section{Measurement of gelation kinetics}

As the polymerization reaction proceeded, the viscosity continuously increased until the full network structure (gel structure) was formed. The gelation time was defined as a period of time for gel formation following the addition of Triethanolamine and measured by a simple tilting method. It was determined by the duration of time taken by the reactant mixture to become viscous and the viscous solution no longer descended in the tilted tube position [11]

\section{Scanning electron microscopy analysis}

Dried SPHCs were cut to expose their inner structure and used for SEM studies. The morphology and porous structures of the SPHCs were examined using ZEISS EVO 18, CARL ZEISS MICROSCOPY (PENTA FET X 3) OXFORD INSTRUMENTS with an operating voltage of $30 \mathrm{kV}$. Images were captured using a digital capture card.

\section{Determination of void fraction}

The void fraction was calculated by the following equation:

$$
\text { Void Fraction }=\frac{\text { Dimensional volume of the hydrogel }}{\text { Total volume of pores }}
$$

The void fraction inside superporous hydrogels was determined by immersing the hydrogels in $\mathrm{HCl}$ solution ( $\mathrm{pH}$ 1.2) up to equilibrium swelling. The dimensions of the swollen hydrogels were measured and by using these data, sample volumes were determined as the dimensional volume. In the meantime, the amount of absorbed buffer into the hydrogels was determined by subtracting the weight of dried hydrogel from the weight of swollen hydrogel and the resulting values were assigned as the total volume of pores in the hydrogels [12].

\section{Water retention}

The following equation was used to determine the water retention capacity (WRt) as a function of time:

$$
W R t=\frac{(W p-W d)}{(W s-W d)}
$$

Where Wd is the weight of the dried hydrogel, Ws is the weight of the fully swollen hydrogel, and $\mathrm{Wp}$ is the weight of the hydrogel at various exposure times. For determination of the water-retention capacity of the hydrogels as a function of the time of exposure at 37 ${ }^{\circ} \mathrm{C}$, the water loss of the fully swollen polymer at timed intervals was determined by gravimetry [13].

\section{Mechanical strength}

Mechanical strength of dried SPH was measured by applying the weight on swelled Superporous hydrogels, until the hydrogels fractured [14].

\section{Density and porosity measurements}

For density measurement, the solvent displacement method was used. Dried SPHCs were used for density measurements, which actually show the apparent densities of the SPHCs. Pieces of SPHCs were taken and weighed in order to determine the mass of each piece. A piece of the polymer was immersed in a predetermined volume of $n$-hexane in a graduated cylinder, and the increase in the volume of $n$-hexane was measured as the volume of the polymer. The density was calculated from the following equation.

$$
\text { Density }=\frac{\mathrm{MSPHC}}{\mathrm{VSPHC}}
$$

Where, VSPHC is the volume of solvent displaced by SPHC and MSPHC is the mass of the SPHC.

For porosity measurement, dried hydrogels were immersed in hexane overnight and weighed after excess hexane on the surface was blotted.

$$
\text { Porosity }=\frac{\mathrm{VP}}{\mathrm{VT}}
$$

Where, VP $=($ VT-VSPHC $)$ is the pore volume of SPHC and VT is the total volume of the SPHC [15].

\section{Water retention}

The following equation was used to determine the water retention capacity.

(WRt) as a function of time of exposure at $37^{\circ} \mathrm{C}$.:

$$
W R t=\frac{(W p-W d)}{W s-W d}
$$

Where, Wd was the weight of the dried hydrogel, Ws was the weight of the fully swollen hydrogel and Wp was the weight of the hydrogel at various exposure times like 15, 45, 90 and $150 \mathrm{~min}$.

\section{Determination of drug content}

For assay of the drug in super porous hydrogels, Atenolol was extracted from $1 \mathrm{~g}$ of each formulation with $20 \mathrm{ml}$ of Methanol for 30 min. The mixture was filtered and drug content was determined using UV-Vis spectrophotometer at $274 \mathrm{~nm}$.

\section{Swelling studies}

A completely dry, pre-weighed, disc-shaped super porous hydrogel was weighed and then immersed in excess of swelling medium (HCl$\mathrm{KCl}$ buffer $\mathrm{pH}$ 1.2). At various time intervals, the hydrogel was removed from the solution and weighed after excessive solution on 
the surface was blotted. Data presented in this experiment were the mean values of triplicate measurements. Results were calculated according to the following equation:

$$
Q=\frac{(M s-M d)}{M d}
$$

Where $Q$ is the swelling ratio, Ms the mass in the swollen state and Md the mass in the dried state. The hydrogels were first swollen in $\mathrm{pH} 1.2 \mathrm{HCl}$ solutions for $30 \mathrm{~min}$ [16].

\section{In vitro release studies:}

In vitro drug release of Atenolol from the superporous hydrogels was evaluated in triplicates at $37 \pm 0.5^{\circ} \mathrm{C}$ using a United States Pharmacopoeia (USP) Dissolution Test Apparatus Type 1 (Rotating Basket Apparatus)) at a rotation speed of $50 \mathrm{rpm}$ in $900 \mathrm{ml}$ of $0.1 \mathrm{M} \mathrm{HCl}(\mathrm{pH} \mathrm{1.2)}$ for $9 \mathrm{~h}$. For this study the capsule filled with hydrogel was kept into the basket and at regular time intervals, $10 \mathrm{ml}$ of the dissolution medium were withdrawn, replaced with an equivalent volume of fresh dissolution fluid and analyzed for the drug using a UV-Vis spectrophotometer (UV-1700, Shimadzu, Japan at $274 \mathrm{~nm}$ [17].

\section{Stability study}

Formulations were subjected to stability testing as per ICH norms. Superporous hydrogel was filled in clean, lacquered, collapsible aluminum tubes, and various replicates were kept at $40{ }^{\circ} \mathrm{C}$ and $75 \%$ RH in a humidity chamber. Superporous hydrogel was assessed for change in appearance, drug content and in vitro release profile at an interval of 7, 15, 30, 60 and $90 \mathrm{~d}$ [18].

\section{RESULTS AND DISCUSSION}

Drug excipient interaction is an important study prior to development of any formulations. Among the various methodologies available to study the drug-excipients interaction, common approaches are-FTIR Spectroscopy, DSC, IR Spectra etc. FTIR Spectroscopy shows the interaction between the molecules at the level of functional groups. Here, drug excipients interaction was done using FTIR Spectroscopy (fig. 1). It was observed from the FTIR Spectra that there may be some physical interactions due to generation of weak bonds as no such shifting of the peaks were marked.

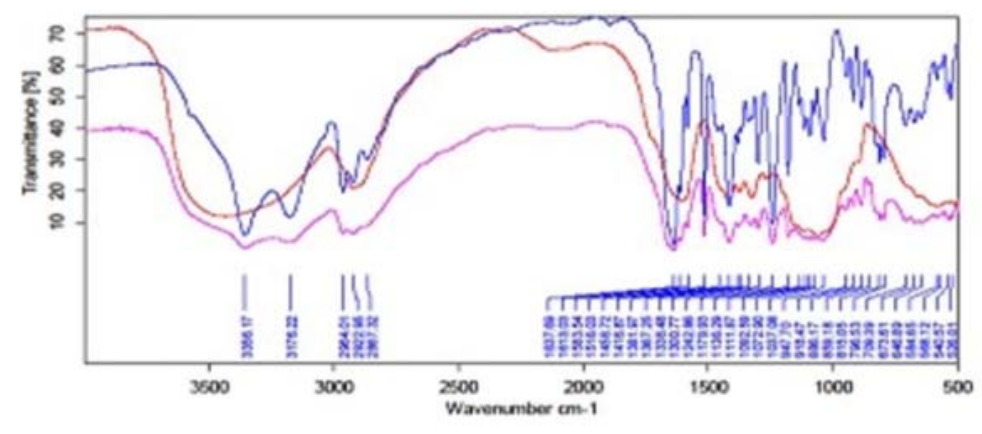

Fig. 1: Overlaid FTIR spectra of drug, mixture of polymers, and mixture of drug with polymers

It is suggested by the FTIR spectra that there may be some physical interactions due to generation of weak to medium intensity bonds as no major shifting of peaks was marked [19]. Mixture of polymers can change the rate of diffusion of drug molecules by changing entanglement in polymeric network. Characteristic bands in FTIR spectrum of drug polymer mixture were seen at $3355.54 \mathrm{~cm}^{-1}$ (the stretching vibration of group- $\mathrm{NH}_{2}$ ), at about $2962 \mathrm{~cm}^{-1}$ (the stretching vibration of $-\mathrm{OH})$ ), Asymmetric and symmetric $\mathrm{C}=\mathrm{O}$ were represented by two bands at $1145 \mathrm{~cm}^{-1}$ (strong) and $1298 \mathrm{~cm}^{-1}$ (weak), respectively.

The time taken for the gelation is same for all the preparations as there was no change in the concentration of Triethanolamine which is responsible for forming hydrogel here. As the polymerization reaction proceeded, the viscosity continuously increased until the full network structure (gel structure) was formed. The gelation time was defined as a period of time for gel formation following the addition of Triethanolamine and measured by a simple tilting method. It was determined by the duration of time taken by the reactant mixture to become viscous and the viscous solution no longer descended in the tilted tube position [20].

Fig. 2-.4. show the SEM pictures of the hydrogel formulation. The picture shows large numbers of pores, indicating that formation of hydrogel with the superporous structure. The scanning electron microscopic photograph of superporous hydrogel (fig. 2-4) clearly shows the presence of pores on the surface. The superporous hydrogel has high porosity and is responsible for faster swelling when compared to conventional hydrogels. The pores are in different size ranges like $1 \mu \mathrm{m}, 2 \mu \mathrm{m}, 10 \mu \mathrm{m}$.

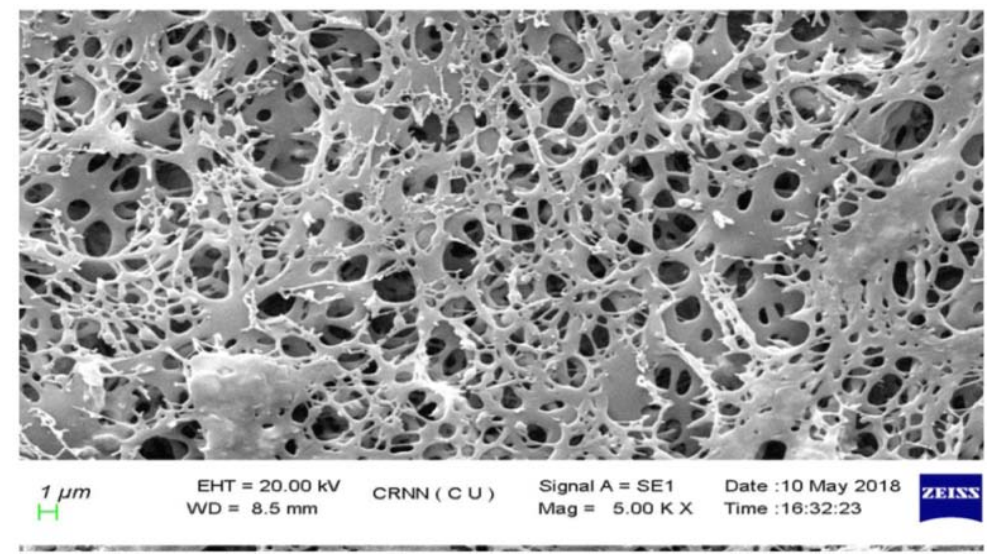

Fig. 2: Scanning electron microscopic photograph of superporous hydrogel at X 5000 of formulation F1 


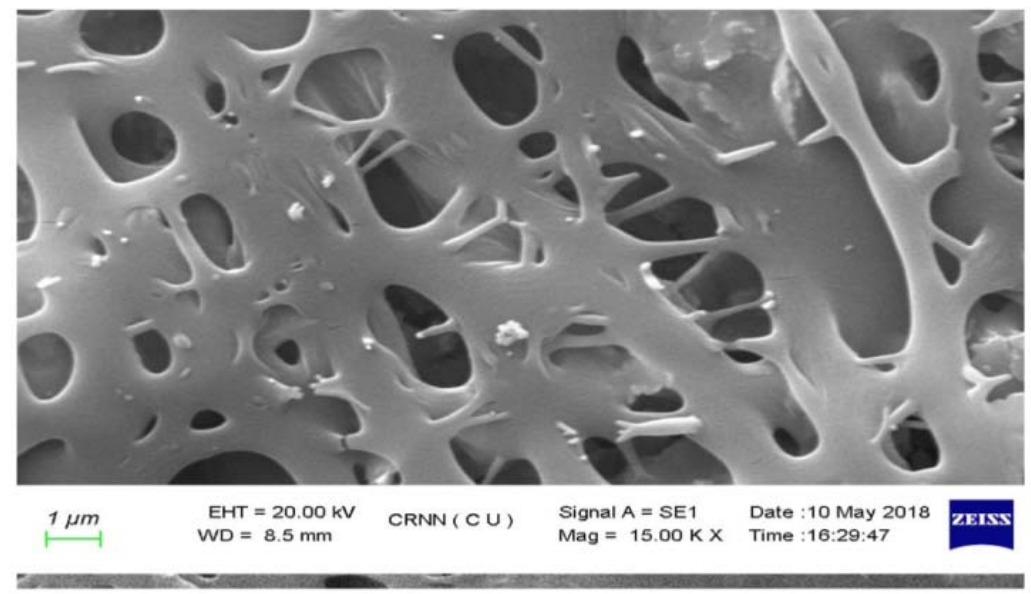

Fig. 3: Scanning electron microscopic photograph of superporous hydrogel at X 15000 of formulation G1

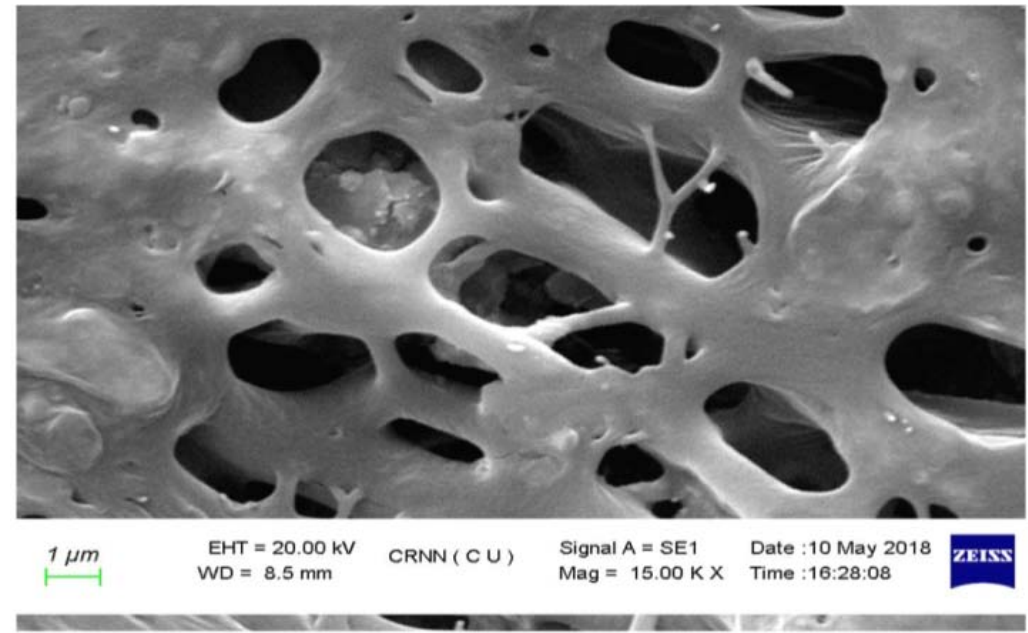

Fig. 4: Scanning electron microscopy photograph of superporous hydrogel at X 15000 of formulation A3

Porosity measurements are shown in fig. 5. The high porosity of the superporous hydrogel is achieved by the addition of pore formers, $\mathrm{NaCl}$ during the polymerization process, resulting in porous structure. The high polar internal surface of these pores within the superporous hydrogels was responsible for the very fast swelling rate, which resulted in a high swelling ratio of the super porous hydrogels.

The superporous hydrogel showed satisfactory physicochemical results (table 2). The range of mechanical strengths (measured in g) was found to be 180 gm-255 gm. For a gastric retention device, the swollen SPH should be strong enough to withstand repeated peristaltic contractions. Thus, it was necessary to improve mechanical property of SPHs. The presence of Ac-Di-Sol increased the cross-linking density of the SPHs by the physical entanglement of the polymer chains with its fibers. When Ac-Di-Sol fibers were added to the solution, they swelled and absorbed the polymer solution. This resulted in physical entanglements of polymer chains through the Ac-Di-Sol fibers. Hence the mechanical load gets shared between Ac-Di-Sol fiber and the polymer structure [21].

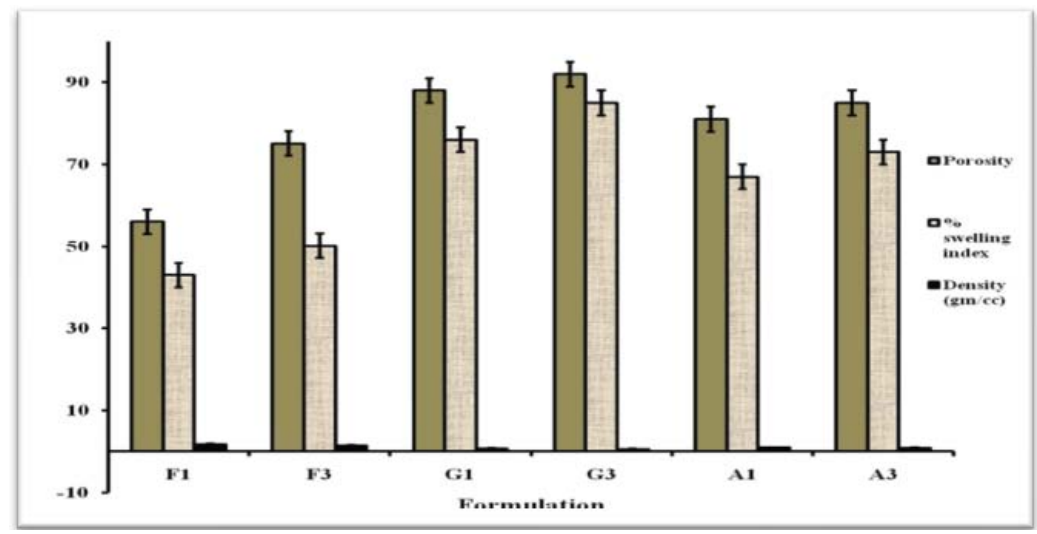

Fig. 5: Comparison of porosity, swelling index and density of the superporous hydrogels $( \pm S D, N=6)$ 
Table 2: Physico-mechanical properties of superporous hydrogel ( $N=6, \pm S D)$

\begin{tabular}{|c|c|c|c|c|c|}
\hline Formulation & Void Fraction & Water retention & Mechanical Strength (gm) & Drug content (\%) & $\%$ Swelling index \\
\hline F1 & $1.161 \pm 0.3$ & $0.575 \pm 0.1$ & $245 \pm 2$ & $98.4 \pm 0.2$ & $43 \pm 1.09$ \\
\hline F3 & $2.19 \pm 0.2$ & $0.846 \pm 0.1$ & $255 \pm 1$ & $99.2 \pm 0.2$ & $50.16 \pm 1.32$ \\
\hline G1 & $2.24 \pm 0.3$ & $0.916 \pm 0.1$ & $200 \pm 2$ & $99.4 \pm 0.2$ & $56.5 \pm 1.16$ \\
\hline G3 & $4.64 \pm 0.3$ & $0.634 \pm 0.1$ & $180 \pm 4$ & $99.2 \pm 0.2$ & $31.3 \pm 1.17$ \\
\hline A1 & $6.26 \pm 0.2$ & $0.864 \pm 0.1$ & $215 \pm 1$ & $99.2 \pm 0.2$ & $34.6 \pm 1.06$ \\
\hline A3 & $5.26 \pm 0.3$ & $0.454 \pm 0.1$ & $220 \pm 2$ & $98.2 \pm 0.2$ & $35.02 \pm 2.56$ \\
\hline
\end{tabular}

The presence of tri ethanolamine increased the overall cross linking density of the superporous hydrogels. This entanglement significantly improved the structural integrity of the hydrogel and decreased stress relaxation, which enhanced its ability to withstand pressure.

The combination of Carbopol and HPMC are responsible for rapid swelling which leads to the interpenetration of the polymer chains at the interface, and improved the buyoency in the gastric environment. Polymer--water interactions are important and serve as the basis for the swelling process in all types of hydrogels. The swelling process itself begins when an SPH is placed in water or other aqueous solutions. This process is first dominated by the attractive forces of the hydrophilic and ionic functional groups in the hydrogel structure. The swelling process continues until each of the functional groups is surrounded by the same amount of water. Next, as the water tries to further dilute the polymer chains, an osmotic effect is created that continues to fill the open pores with water until opposed by the contractive forces of the crosslinked hydrogel structure [22].

In vitro drug release data of all the formulations was subjected to goodness of fit test by linear regression analysis according to zero order and Higuchi and Korsmeyer-Peppas models to ascertain the mechanism of drug release. The results of linear regression analysis including rate constants and regression coefficients are summarized in table 3.

To investigate the drug release mechanism, the data were evaluated by kinetic models representing zero-order (fig. 6), Higuchi and Korsmeyer Peppas [23]. The cumulative percent of drug released versus time plot exhibits curvilinear nature, which suggests that the drug release is not governed by zero order kinetics. This observation is confirmed by fitting the dissolution data to zero-order model where comparatively low values of correlation coefficients $\left(\mathrm{R}^{2}\right)$ are obtained. The results of above the mentioned studies show that drug release from the superporous hydrogels are much more acquainted with Korsmeyer-Peppas, Kopcha, and Higuchi models. The experimental data were fitted to Korsmeyer-Peppas exponential equation $\mathrm{Mt} / \mathrm{M} \alpha=\mathrm{Ktn},[\mathrm{Mt} / \mathrm{M} \alpha=$ the fractional drug release into the dissolution medium, $\mathrm{K}=\mathrm{a}$ constant, $\mathrm{n}=$ diffusional exponent] [24]. In the present study it was observed (table 2) that the $n$ varies from 0.3 to 0.5 representing change in drug transport mechanism. It is due to the changes in polymeric ratio. Among the six batches drug release from is fully governed by quasi Fickian and Fickian diffusion ( $\mathrm{n}<0.5$ and 0.5 respectively).

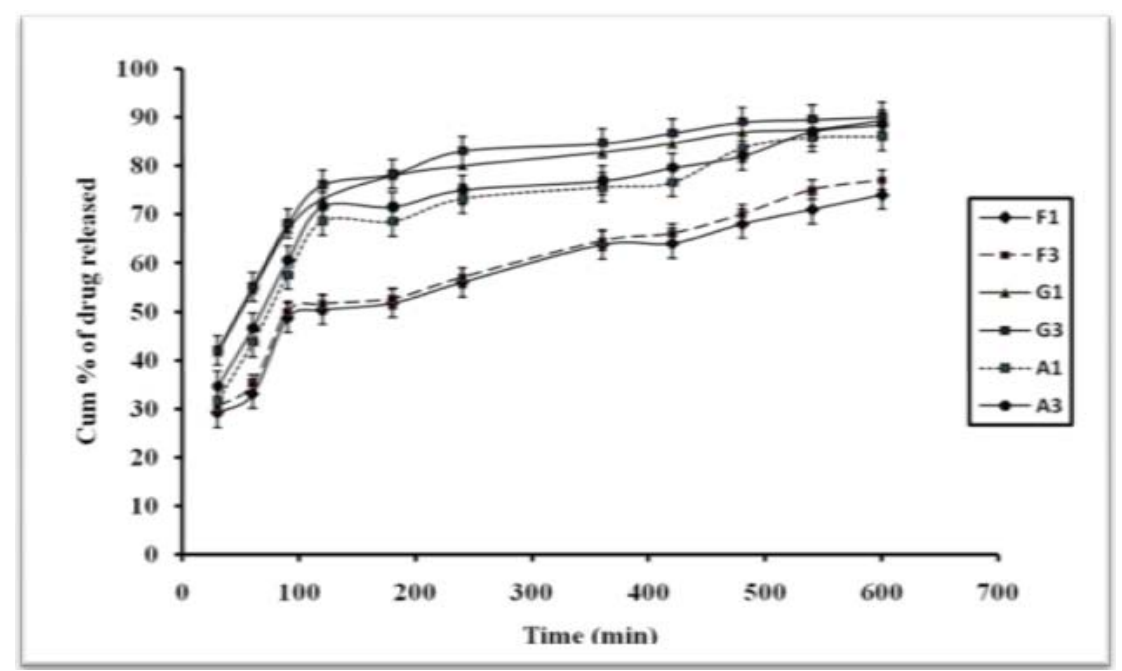

Fig. 6: Drug release profile of the formulations in buffer pH-1.2 $( \pm \mathrm{SD}, \mathrm{N}=6)$

For further clarification Kopcha mathematical model was considered. This result is supported by the determination of the ratios of the exponents $\mathrm{A} / \mathrm{B}$ (i.e., diffusional factor $\mathrm{A}$ and erosional factor B) [25]. The ratios (A/B) were greater than 1 in all the batches. In case of F1, F3 and G1, G3 (table 3) the value of the release exponent, $n$, where $<0.45$ indicating that Atenolol release was controlled not only by diffusion. The diffusion rate of a drug depends on the physical structure of the polymer network and its chemical nature. When the formulation gets fully hydrated, drug diffusion occurs through the pores present. In gels where lower hydration occurs, the drug is dissolved in the polymer and is transported between the chains. Cross-linking of the polymers is responsible for increasing the hydrophobicity of the gel formed at the outer surface and reduces the diffusion rate of the drug [26]. For further confirmation, fitting the data to the Kopcha model showed the evidence of drug release by a combination of diffusioncontrolled and chain relaxation-swelling mechanism. In case of A1, A2 due to presence of combination of HPMCK100M and HPMC $\mathrm{K} 15 \mathrm{M}$ the gel became more hydrophobic and retarded the diffusion of drug from the superporous hydrogel. The drug release profile may be easily related to the swelling properties of these superporous hydrogels. This study demonstrates that superporous hydrogels of Atenolol may be suitable for use as a gastro retentive drug delivery system. 
Table 3: Kinetics-data of drug release from superporous hydrogel

\begin{tabular}{|c|c|c|c|c|c|c|c|c|c|c|}
\hline \multirow[t]{2}{*}{ Formulation } & \multicolumn{2}{|c|}{ Zero order } & \multicolumn{2}{|c|}{ Higuchi kinetics } & \multicolumn{2}{|c|}{ Korsmeyer-peppas } & \multicolumn{4}{|c|}{ Kopcha model } \\
\hline & $\mathbf{R}^{2}$ & $\mathbf{K}_{1}$ & $\mathbf{R}^{2}$ & $\mathbf{K}_{3}$ & $\mathbf{R}^{2}$ & n & $\mathbf{R}^{2}$ & $\mathbf{A}$ & B & A/B \\
\hline F1 & 0.881 & 0.067 & 0.943 & 2.15 & 0.911 & 0.32 & 0.984 & 5.88 & 0.131 & 44.88 \\
\hline F3 & 0.903 & 0.070 & 0.952 & 2.241 & 0.891 & 0.319 & 0.987 & 6.155 & 0.140 & 43.85 \\
\hline G1 & 0.721 & 0.062 & 0.832 & 4.16 & 0.994 & 0.426 & 0.998 & 8.871 & 0.223 & 39.77 \\
\hline G3 & 0.702 & 0.063 & 0.815 & 4.25 & 0.993 & 0.433 & 0.997 & 8.94 & 0.220 & 40.63 \\
\hline A1 & 0.764 & 0.074 & 0.869 & 4.925 & 0.991 & 0.559 & 0.985 & 6.654 & 0.118 & 56.38 \\
\hline A3 & 0.746 & 0.070 & 0.848 & 2.332 & 0.990 & 0.524 & 0.989 & 7.323 & 0.149 & 49.14 \\
\hline
\end{tabular}

${ }^{*} \mathrm{~K} 1, \mathrm{~K} 3$ : Release rate constant, n: Release exponent (indicative of drug release mechanism), A and B: diffusional factor and erosional factor constants

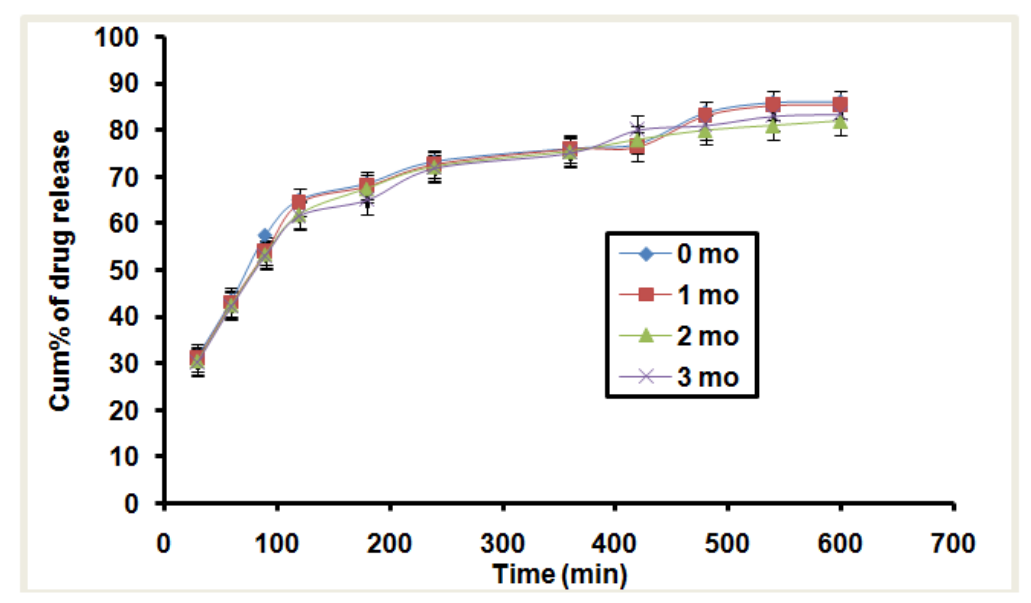

Fig. 7: Drug release profile from the formulations in buffer pH-1.2 in respect to stability study at 1, 2 1nd 3 mo interval

During stability studies, formulation appeared to be off white, hard. It was also noted from the studies that there were no considerable changes in drug content as well as the cumulative percent of drug release. Therefore, no evidence of degradation of drug was noted.

Drug release profiles prior and after $3 \mathrm{mo}^{\prime}$ stability study of formulation A1, similarity factor $\left(f_{2}\right)$ was calculated (fig. 7). Similarity factor was found to be $\mathrm{f}_{2}=65.33(>50)$. As similarity factor greater than 50 indicates the good stability of the product, in view of this it has been concluded that the formulation was stable over the period of 3 mo [27].

\section{CONCLUSION}

Controlled drug delivery via the polymer based systems has been successful to be prevailing both in the present and in future; as having numerous potential advantages for scientific as well as economic reasons. The thought behind developing Superporous hydrogel was to deliver Atenolol in the gastric environment in a continual manner for extended time periods to reduce the application frequency, hypersensitive reactions and to improve bioavailability, safety than marketed conventional formulation.

HPMCK100M, HPMCK15M, Carbopol 971p have been employed for the formulations. $\mathrm{NaCl}$ was introduced as pore formers and Ac-DiSol to increase the physical cross linking of polymer chains to fix it. SEM photographs of the formulations clearly indicated the formation of a large number of pores confirming it as superporous hydrogel. A significant variation is observed in the in vitro release pattern of Atenolol from the SPH in relation to change the ratio of two grades of HPMC to Carbopol 971p. There was variation in transport mechanism from quasi diffusion to anomalous by changing the ratio of HPMCK100M and HPMCK15M. In vivo studies are another future prospect to get a clear picture of IVIVC.

\section{ACKNOWLEDGEMENT}

The authors wish to thank NSHM Knowledge Campus, Kolkata, India for their valuable support for this research.

\section{AUTHORS CONTRIBUTIONS}

All the authors have contributed equally

\section{CONFLICT OF INTERESTS}

Declared none

\section{REFERENCES}

1. Allan SH. Hydrogels for biomedical applications. Adv Drug Delivery Rev 2002;43:3-12.

2. Chen J, Park H, Park K. Synthesis of superporous hydrogels: hydrogels with fast swelling and superabsorbent properties. J Biomed Mater Res 1999;44:53-62.

3. Yong Q, Park K. Superporous IPN hydrogels having enhanced mechanical properties. AAPS PharmSciTech 2003;4:406-12.

4. Reddy AM, Narsimharao N, Srinivasababu P, Navyasri N, Reddy AA. Formulation and characterization of super porous hydrogel of Nizatidine. Indian J Res Pharm Biotech 2015;3:341-50.

5. Chen J, Park K. Synthesis and characterization of superporous hydrogels composites. J Controlled Release 2000;65:73-82.

6. Sharma PK, Asthana GS, Asthana A. Hydrogel advancement: a new approach for gastroretentive drug delivery. Int J Pharm Clin 2016;8:1418-22.

7. Akhtar MF, Hanif M, Ranjha NM. Methods of synthesis of hydrogels, a review. Saudi Pharml J 2016;24:554-6.

8. Niharika MG, Krishnamoorthy K, Akkala M. Overview on floating drug delivery system. Int J Appl Pharm 2018;10:65-71.

9. Vadia N, Rajput AS. Mesoporous material, mcm 41: a new drug carrier. Asian J Pharm Clin Res 2011;4:44-53.

10. Dantas MGB, Reis SA, Damasceno GB, Damasceno CMD. Development and evaluation of stability of a gel formulation containing the monoterpene borneol. Sci World J 2016. Doi:10.1155/2016/7394685

11. Farid AD, Verhoef JC, Borchard G, Rafiee MT, Hans EJ. Development and characterization of a novel peroral peptide drug delivery system. J Controlled Release 2001;71:307-18. 
12. Köse GT, Kenar H, Hasirci N, Hasirci V. Macroporous poly (3hydroxybutyrate-co-3-hydroxyvalerate) matrices for bone tissue engineering. Biomaterials 2003;24:1949-58.

13. Gupta NV, Shivakumar HG. Preparation and characterization of superporous hydrogels as gastroretentive drug delivery system for rosiglitazone maleate. DARU 2010;18:200-10.

14. Deore LP, Devidas GB. Superporous hydrogel (SPH): an innovative approach of gastro retention. Res J Pharm Bio Chem Sci 2013;4:261-75.

15. Chavda HV, Patel CN. Preparation and characterization of swellable polymer-based superporous hydrogel composite of poly (Acrylamide-co-Acrylic Acid). Trends Biomater Artif Organs 2010;24:83-9.

16. Majee SB, Avlani D, Biswas GR. HPMC as capsule shell material: physicochemical, pharmaceutical and biopharmaceutical properties. Int J Pharm Pharm Sci 2017;9:1-6.

17. Rajab NA, Jawad MS. Formulation and in vitro evaluation of piroxicam microsponge as a tablet. Int J Pharm Pharm Sci 2015;8:104-10.

18. Muthuswamy R, Deepan D. Formulation and evaluation of superporous hydrogel tablet rabeprazole sodium as gastroretentive drug. Eur J Pharm Med Res 2018;5:271-8.

19. Mukherjee B, Das S, Patra B, Layek B. Nefopam containing transdermal-matrix patches based on pressure-sensitive adhesive polymers. PharmTech 2006;30:146-63.
20. Bonardi AH, Bonardi F, Morlet S. Photoinduced thermal polymerization reactions. Macromolecules 2018 Doi:10.1021/acs.macromol.8b01741

21. Kumar A, Pandey M, Mamman K, Saraf S. Synthesis of fast swelling superporous hydrogel: effect of concentration of crosslinker and acdisol on swelling ratio and mechanical strength. Int J Drug Delivery 2011;2:135-5.

22. Naziha C, Lhocine Y, Lukas G, Federico LM, Soumia C, Silvia F. History and applications of hydrogels. J Biomed Sci 2015;4:1.

23. Hebbar S, Dubey A, Ravi GS, Mascarenhas SB. Studies on crosslinked chitosan hydrogel for matrix tablets of montelukast sodium. Int J Appl Pharm 2017;9:22-7.

24. Babu BR, Kilaru NB. An unified mathematical expression for ideal peppas model: prospective estimation of percent deviation. Asian J Pharm Tech 2016;6:189-7.

25. Kopcha M, Lordi NG, Tojo KJ. Evaluation of release from selected thermo softening vehicles. J Pharm Pharmacol 1999;43:382-7.

26. Kamaly N, Yameen B, Wu J, Farokhzad OC. Degradable controlled-release polymers and polymeric nanoparticles: mechanisms of controlling drug release. Chem Rev 2016;116:2602-63.

27. Gohel MC, Ramkishan A, Patel TM, Pandya R, Suthar V, Koradia $\mathrm{H}$, et al. Nomogram for computing the value of similarity factor. Indian J Pharm Sci 2014;76:245-51. 